\title{
Analyzing Virtual Reference Station for GPS surveying: experiments and applications in a test site of the northern Apennines (Italy)
}

\author{
Arianna Pesci $\left({ }^{1}\right)$, Fabiana Loddo $\left({ }^{1}\right)$, Nicola Cenni $\left({ }^{2}\right)$, Giordano Teza $\left({ }^{3}\right)$ and Giuseppe Casula $\left({ }^{1}\right)$ \\ ( $\left.{ }^{1}\right)$ Istituto Nazionale di Geofisica e Vulcanologia, Sezione di Bologna, Italy \\ ${ }^{(2)}$ Dipartimento di Fisica, Settore Geofisica, Università degli Studi di Bologna, Italy \\ ${ }^{(3)}$ Dipartimento di Geoscienze, Università degli Studi di Padova, Italy
}

\begin{abstract}
The availability of a GPS network of 10-20 km mean size, provides good topographical support for the measurement of ground displacements, even at a local scale such as a landslide. In particular, a series of multitemporal kinematic or rapid-static GPS acquisitions of a landslide allows a good characterization of its displacements if the measurements are referred to a GPS reference network. Nevertheless, a wider network formed by stations located at long distances, for example at several tens of kilometers, characterized by large spacing, can lead to results affected by high noise, degrading the accuracy of final point positions. In order to obtain an adequate GPS reference network, some virtual reference stations (VRSs) can be introduced, even if a network refinement based on VRS cannot reach the same accuracy of a real local network. Some experiments, including measurements on a real landslide, have been performed in order to evaluate the performance of this technique. The results point out that the standard deviation of the obtained solutions is about two or three times larger than those which can be reached using a real local network.
\end{abstract}

Key words GPS - Virtual Reference Stations Rapid Static Survey - Landslide Monitoring

\section{Introduction}

The global positioning system (GPS), used in static and kinematic mode, provides highly accurate estimations of measured point coordinates and therefore is widely applied for the

Mailing address: Dr. Arianna Pesci, Istituto Nazionale di Geofisica e Vulcanologia, Sezione di Bologna, Via Donato Creti 12, 40128 Bologna, Italy; tel. +39 0514151416; fax +39 0514151498; e-mail: pesci@bo.ingv.it monitoring of slow or fast ground surface deformations of the Earth's crust. Information provided by a GPS network can be used to evaluate the regional tectonics (see for example, Cenni et al. 2008 or the local seismogenic structures (Hunstad et al., 1999; Pietrantonio and Riguzzi, 2004; Serpelloni et al., 2001; Anzidei et al., 2005; Pesci and Teza, 2007). At the scale of a landslide, GPS data can be used to monitor gravitational macroscopic effects due to rock-mass collapses, landslide activations or other instability effects (Gili et al., 2000; Moss, 2000; Bonforte et al., 2002; Mora et al., 2003; Tzenkov and Gospodinov, 2003; Squarzoni et al., 2005; Pesci et al., 2005; Baldi et al., 2008).

Despite the fact that higher precision is achieved by static GPS applications, GPS kinematic surveying (Rizos and Han, 1998) can al- 
so provide highly accurate trajectories, produced by a rover station which moves throughout measurement sessions. This technique can be useful either to define the correct position of photogrammetric camera installed on aircraft platforms (Beutler et al., 1995; Achilli et al., 1997; Baldi et al., 2000), or to acquire a large number of points distributed on a physical surface when fast and accurate monitoring is required. In the latter case a point cloud, i.e. a set of coordinates of trajectory points, is obtained, with a nominal accuracy of a few centimeters (or less) in the horizontal components, while the error of the vertical component is generally two or three times greater than the planimetric one. Some tests are reported in Pesci et al. (2005), showing the statistical data distribution of coordinate solutions for kinematic applications in different surveying modes; crossover analyses were used to evaluate solution reliability when the GPS rover was moving or stationary throughout the measurement session. For continuous kinematic surveys, when the antenna is moved on the ground following a planned trajectory to cover the area with a large and dense irregular grid, the human error induced in point positioning by the operator has to be considered due to the fact that the measured points are referred to the topographic surface (Pesci et al., 2004).

The final coordinates of GPS points depend on many factors during data processing, i.e. network geometry, baseline length, local geometry and shape of the monitored area and visibility of the satellite constellation, atmospheric effects, computation strategy, etc. (HofmanWellenhof et al., 1997).

A common and widely used approach for GPS surveying and processing requires three or more reference stations as datum of an external reference system, a set of fiducial stations inside and at least two stations (base and rover) working in the specific investigated area; the number of these stations is defined on the basis of the characteristics of the studied area (see e.g. HofmannWellenhof et al., 1998). In particular, the large network is mainly adopted to register the solution into a common reference frame, the internal fiducial stations provide a solid geometry for robust data processing and the latter can also be ac- quired in kinematic or fast/static mode, to collect coordinates of points at the surface or specific natural or artificial targets. In order to obtain good data, the mean spacing of the external network should be no more than $10-20 \mathrm{~km}$ because a line-length dependent uncertainty of 0.5-1 ppm is added to the base positional uncertainty for each component. Such a line-length dependent error is caused mostly by errors in the orbital ephemeris data and atmospheric effects. Nevertheless, despite the fact that the Italian territory is widely covered by continuously operating permanent GPS stations (see e.g. Vespe et al., 2000; Anzidei et al., 2006), their mean spacing generally ranges from $40 \mathrm{~km}$ to $100 \mathrm{~km}$, and several other stations would be necessary for monitoring purposes. On the other hand, the increase in the number of GPS permanent stations to reduce the network mean spacing, is not always possible and is very expensive. Some public and private companies, often interested in topographical applications, work to make the GPS permanent network denser and regularly distributed on the territory. Some Italian regions (see for example the Autonomous Region of Friuli Venezia-Giulia: http://www.crs.inogs.it/frednet/immagini/_notes/ Ghidini.pdf and the Emilia Romagna Region, where both INGV, ASSOGEO and University GPS receivers are present) are now covered by a high number of stations; nevertheless, this good status is not present in other regions where the mean spacing between the stations is rarely smaller than $50 \mathrm{~km}$.

Recently, a new approach has been introduced to solve the problems related to the availability of the permanent stations. It is based on the generation of synthetic data at a chosen and defined coordinate point, called Virtual Reference Station (VRS, Higgins, 2002; Häkli, 2004; $\mathrm{Hu}, 2005)$. Thanks to VRS introduction, the mesh of reference stations can be adequately refined, and a step of a few $\mathrm{km}$ can be obtained avoiding the introduction of new real stations.

This article reports the results of some experiments carried out to test the accuracy of the solutions which can be achieved from VRS data post-processing, and provide the VRS methodology for fast kinematic surveys, in areas subjected to high surface deformations and terrain instabilities, in particular landslides. The 
work is based on three key points to validate VRS methodology for monitoring proposal:

i) investigation of possible systematic effects in the estimation of point coordinates;

ii) test of the solution reliability and internal accuracy;

iii) definition of a methodology useful in providing solution correctness during kinematic continuous surveying for topographic surface acquisition and modeling.

\section{VRS basics}

A VRS implies a GPS network formed of at least three stations, used to generate a synthetic dual-frequency code and carrier-phase data at a virtual base located in a defined point of known or given coordinates. The VRS concept is to create one or more synthetic GPS stations close to user's receivers (rover) and to generate the corresponding RINEX files (Gurtner, 1994) virtually belonging to the chosen physical points. In this way, the user can obtain a large amount of data, related to real and virtual stations, and can therefore obtain robust solutions (Higgins, 2002; Häkli, 2004; Hu, 2005).

Using this technique, data provided by real active permanent stations are used as a basis for the estimation of synthetic code and phase observables of the GPS signal in a position near the survey field whereas the standard approach (RTK surveys) should be based on transmission of the data related to the nearest permanent station to the user, whose distance from the interesting point could be several tens of $\mathrm{km}$. Thanks to the very short distance between the virtual station and the GPS receiver used in the in-field measurements (i.e., the rover), the linelength dependent error in the data post-processing can be reduced, even if the ppm error in the case of a VRS is clearly greater than the one related to a real permanent station.

The VRS solutions can be achieved in nearreal-time (akin to real-time kinematic, RTK) or in post-processing mode, depending on the user's needs. The common RTK approach requires high-bandwidth telecommunications among the reference stations, the master control station (where the virtual GPS data are comput- ed) and are subsequently transmitted to the user. This allows the rovers to compare their own phase measurements with the ones received from the near virtual base station and to calculate their relative position with respect to the base. The distances imposed by conventional RTK applications are typically of the order of 10-20 km.

The VRS approach does not require a physical reference station close to the user, but a multi-reference station (MRS) network surrounding the area of interest, allowing the user to access data from a virtual GPS reference station at any interpolated location within the area covered by the network ( $\mathrm{Hu}, 2003)$. A multistep approach is commonly used to compute VRS data, as follows: data transfer from the reference network to a computing centre; use of network data in order to model errors related to ionospheric and tropospheric signal propagation as well as to satellite orbits; fixing of carrier phase ambiguities for the network baselines; derivation of actual errors (centimeter accuracy) on the baselines using the fixed carrier phase observations; application of error models (linear or more sophisticated) to predict the errors at the user location and the virtual reference file creation at the chosen point. Finally, the virtual data can be transmitted to the user in standard formats (RTCM) during real time measurements or can be used in post-processing and stored for specific applications (Vollath et al., 2000).

The capability to generate synthetic data allows the user to work in post-processing, using both VRS and rover (real) data, overcoming the distances imposed by conventional RTK and nominally reaching an accuracy of a few centimeters $(1-3 \mathrm{~cm})$ for point coordinate solutions, depending on the inter-distances between reference stations that usually do not exceed 50-70 km (Wanninger, 2002).

The GPS industries have developed software packages able to generate synthetic RINEX starting from data belonging to at least three GPS stations. In order to process the GPS data for the following experimentations, according to the standard data analysis procedures, Trimble Total Control (TTC) software has been used. This is a computationally efficient, powerful, easy-to-use commercial software package, capable of pro- 
cessing GPS and topographic data. In particular, TTC provides advanced geodetic control and GPS processing and analysis tools, including: automatic internet download of GPS and control data, GPS and GLONASS (GLObal NAvigation Satellite System) baseline processing, data processing for static, rapid static (or fast-static), kinematic and continuous surveys, geodetic network adjustment, geodetic transformations, analysis of residual error distribution and Virtual Reference Station (VRS) post-processing (http://www.hydronav.com/images/landsurvey/p df/TotalControlTN.pdf).

\section{The experimental network and VRS generation}

The GPS network used for computations is formed of four stations (MO01, MO02, MO05, BO03) belonging to the ASSOGEO network (see Trimble Geomatics and Engineering Division, http://www.assogeo.net), with baselines of $\sim 50 \mathrm{~km}$. The stations are equipped with
Trimble receiver/antenna instruments (Trimble, 1999). Another station (TMP1) with similar instruments is added to provide a more correct network geometry, while the user's stations (STA1, STA2) are installed on the roof of the Department of Physics (Bologna University), at a distance of about $10 \mathrm{~m}$ from each other. The used network is shown in fig. 1 .

The RINEX files of all the available stations are loaded into the TTC importing box and arranged for the processing. The IGS accurate ephemeris (Kouba, 2001) are downloaded and imported into the current project together with the ionospheric model provided by CDDIS archives (Crustal Dynamics Data Information System, ftp://cddis.gsfc.nasa.gov/pub/gps). Data processing starts with the definition of phaseobservables forming double differences with L2 or with a linear combination of L1 and L2 GPS frequencies, for distances longer than 5 $\mathrm{km}$.

The post-processing procedure is iterative. After a first network processing, the data are adjusted to improve the solution accuracy. In
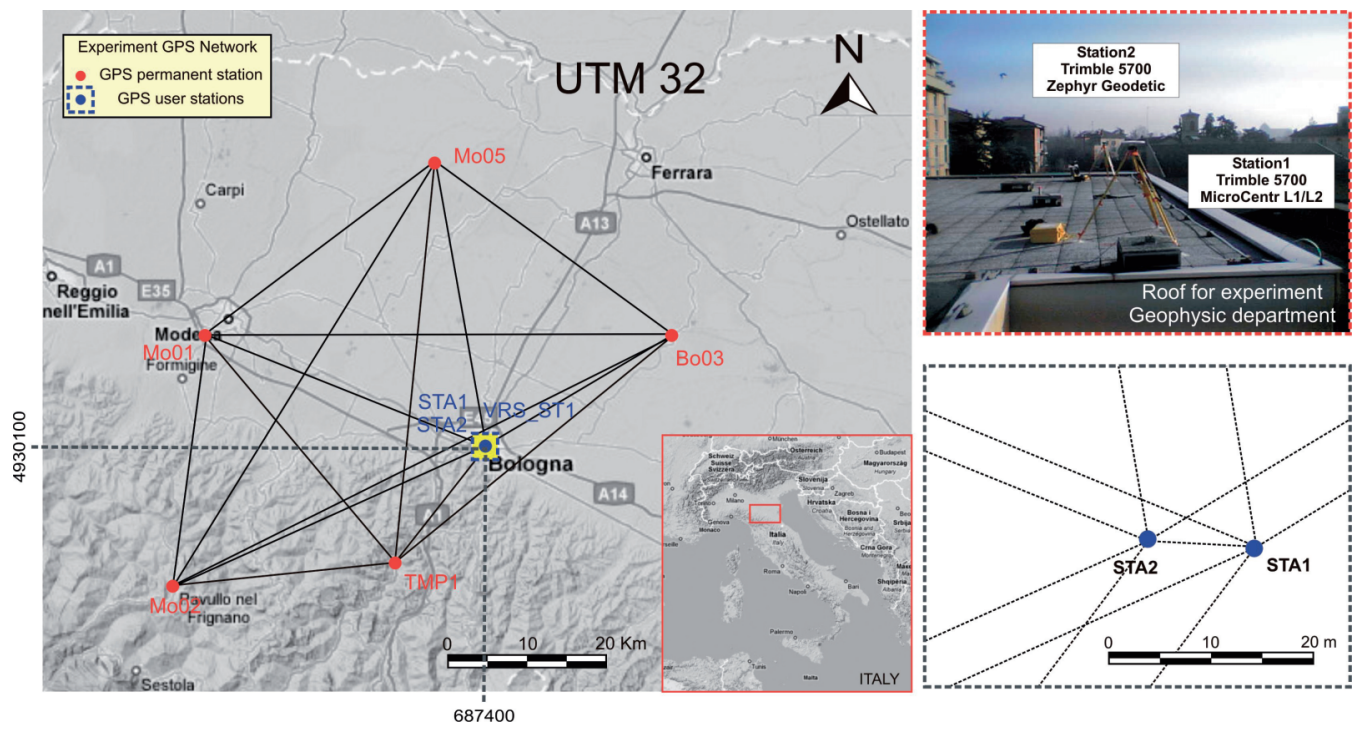

Fig. 1. Scheme of the GPS network. Four Assogeo stations (BO03, MO01, MO02 and MO05), a temporary TMP1 station equipped with Trimble receiver and the two Trimble stations installed on the University roof. 
Table I. Results from processing and network adjustment: station coordinates in UTM and errors at 2 sigma level. The vertical components refers to elevation (WGS84), no a geoidic model is used.

\begin{tabular}{lcccccc}
\hline \hline \multicolumn{7}{c}{ First network processing and adjustment } \\
\hline Point & North $(\mathbf{m})$ & $\sigma(\mathbf{m m})$ & East $(\mathbf{m})$ & $\sigma(\mathbf{m m})$ & Up $(\mathbf{m})$ & $\sigma(\mathbf{m m})$ \\
\hline BO03 & 4944745.713 & 1.2 & 711693.753 & 1.0 & 52.469 & 1.5 \\
MO01 & 4944799.698 & 1.2 & 650660.832 & 1.0 & 95.738 & 1.4 \\
MO02 & 4911304.665 & 1.2 & 646240.539 & 1.0 & 740.959 & 1.5 \\
MO05 & 4967530.173 & 1.2 & 680663.459 & 1.0 & 64.394 & 1.4 \\
TMP1 & 4915717.101 & 1.1 & 675989.848 & 0.9 & 453.998 & 1.8 \\
STA1 & 4930119.897 & 1.1 & 687409.317 & 0.9 & 99.682 & 1.6 \\
STA2 & 4930120.395 & 1.1 & 687398.746 & 0.9 & 99.698 & 1.3 \\
\hline
\end{tabular}

the first processing, only the BO03 station is kept fixed at its known coordinates to constrain the network, avoiding baselines deformation. Therefore, the estimates of coordinates for MO01, MO02, MO05, TMP1, STA1 and STA2 stations into the UTM $(32 \mathrm{~N})$ reference frame are obtained, together with their error ellipses at $1-\sigma$ level (fig. 1). The processing of differential GPS data provides very accurate solutions, and the baselines are obtained with an error of a few millimeters (concerning relative solutions), related to differential data. The final solution was obtained by means of adjusting procedures, generally adopted to provide a single coordinate result in a chosen reference frame. In this case, a first adjustment was performed starting from the first network solution leading to a reference coordinate data set. Subsequently, a second adjustment was done, fixing all the stations on their known coordinates, with the exception of the rovers STA1 and STA2. The solutions, in terms of point coordinates and errors are listed in table I. Errors are given at $1-\sigma$ confidence level.

The VRS module of TTC allows the generation of a RINEX file related to a virtual station with a chosen sampling rate. At the STA1 point VRS is generated at a $30 \mathrm{~s}$ sampling rate. This virtual station, called VRS_ST1, is created imposing the previously estimated coordinates, that is the data related to the permanent station of the used GPS network. The GPS data are then processed using the same procedure described above (preliminary network processing and adjustment), and the related results are listed in table II. Table III shows the residuals, that is the differences between the solutions computed in the two cases for each station. In this table, the coordinate differences of STA1 with respect to the synthetic VRS_ST1 are in bold.

\section{Test of internal VRS accuracy and solution stability}

In order to evaluate the internal accuracy and the solution stability of the VRS-based approach in kinematic mode, another experiment was carried out. In this case, data processing was performed considering both the STA1 and STA2 stations, where STA1 is used as base station and STA2 as a rover. The coordinates of STA1 are fixed on the data previously estimated, whereas the STA2 data are processed in kinematic mode using the On-The-Fly (OTF) algorithm (Landau and Euler, 1992), which is available in the TTC module, providing a coordinate for each measurement epoch (in this case $30 \mathrm{~s}$ ). The experiment was divided into two phases. In the first, the processing of STA2 da- 
Table II. Processing results using the virtual station instead of STA1 and network adjustment at $2-\sigma$ confidence level; station coordinates in UTM and errors at $1-\sigma$ level. The vertical components refers to ellipsoidal elevation (WGS84), no geoid model is used.

\begin{tabular}{lcccccc}
\hline \hline \multicolumn{7}{c}{ Second computation and adjustment } \\
\hline Point & North $(\mathbf{m})$ & $\sigma(\mathbf{m m})$ & East $(\mathbf{m})$ & $\sigma(\mathbf{m m})$ & Up $(\mathbf{m})$ & $\sigma(\mathbf{m m})$ \\
\hline BO03 & 4944745.713 & 1.2 & 711693.753 & 1.1 & 52.473 & 1.5 \\
MO01 & 4944799.698 & 1.1 & 650660.831 & 1.2 & 95.742 & 1.5 \\
MO02 & 4911304.666 & 1.2 & 646240.540 & 1.0 & 740.962 & 1.6 \\
MO05 & 4967530.176 & 1.3 & 680663.460 & 1.0 & 64.399 & 1.6 \\
TMP1 & 4915717.102 & 1.2 & 675989.848 & 1.0 & 454.000 & 1.8 \\
VRS_ST1 & 4930119.898 & 1.2 & 687409.320 & 1.0 & 99.689 & 1.6 \\
\hline
\end{tabular}

Table III. Coordinate residuals obtained by direct coordinate differences, computed in the same reference frame.

\begin{tabular}{lccc}
\hline \hline \multicolumn{4}{c}{ Coordinate residuals } \\
\hline \hline Point & $\Delta$ North $(\mathbf{m m})$ & $\Delta$ East $(\mathbf{m m})$ & $\Delta$ Up $(\mathbf{m m})$ \\
\hline BO03 & 0.0 & 0.0 & -5.0 \\
MO01 & 0.0 & 1.0 & -4.0 \\
MO02 & -2.0 & -1.0 & -3.0 \\
MO05 & -3.0 & -2.0 & -5.00 \\
TMP1 & -1.0 & 1.0 & -2.0 \\
ST1-VRS ST1 & 0.0 & -2.0 & -6.0 \\
\hline
\end{tabular}

ta was performed considering the real STA1 station, whereas VRS_ST1 was considered in the second. In both cases, the same procedures and settings were adopted. The experiment was completed by a comparison between the corresponding solutions.

In both the phases, the obtained kinematic point cloud describes the trajectory of a rover station (STA2) staying on the same point throughout the measurement session. In this way, the dispersion of the points can be easily evaluated, as shown in fig. $2 \mathrm{a}$. The results show that if STA1 is used, the point cloud is charac- terized by low noise, with horizontal deviations of about $0.005 \mathrm{~m}$ and a vertical deviation of about 0.008. If VRS_ST1 is used instead of ST1, the results are characterized by a higher noise level for both the horizontal and vertical components $\mathrm{m}$ (see figs. 2a-d), leading to standard deviations of about $0.011 \mathrm{~m}$ and $0.025 \mathrm{~m}$ respectively, as shown in fig. 3. Even if a small number of outliers exists, the obtained results are in agreement.

This simple experiment tests the effects on the internal precision of the GPS solutions using a virtual station. In particular, the statistical 


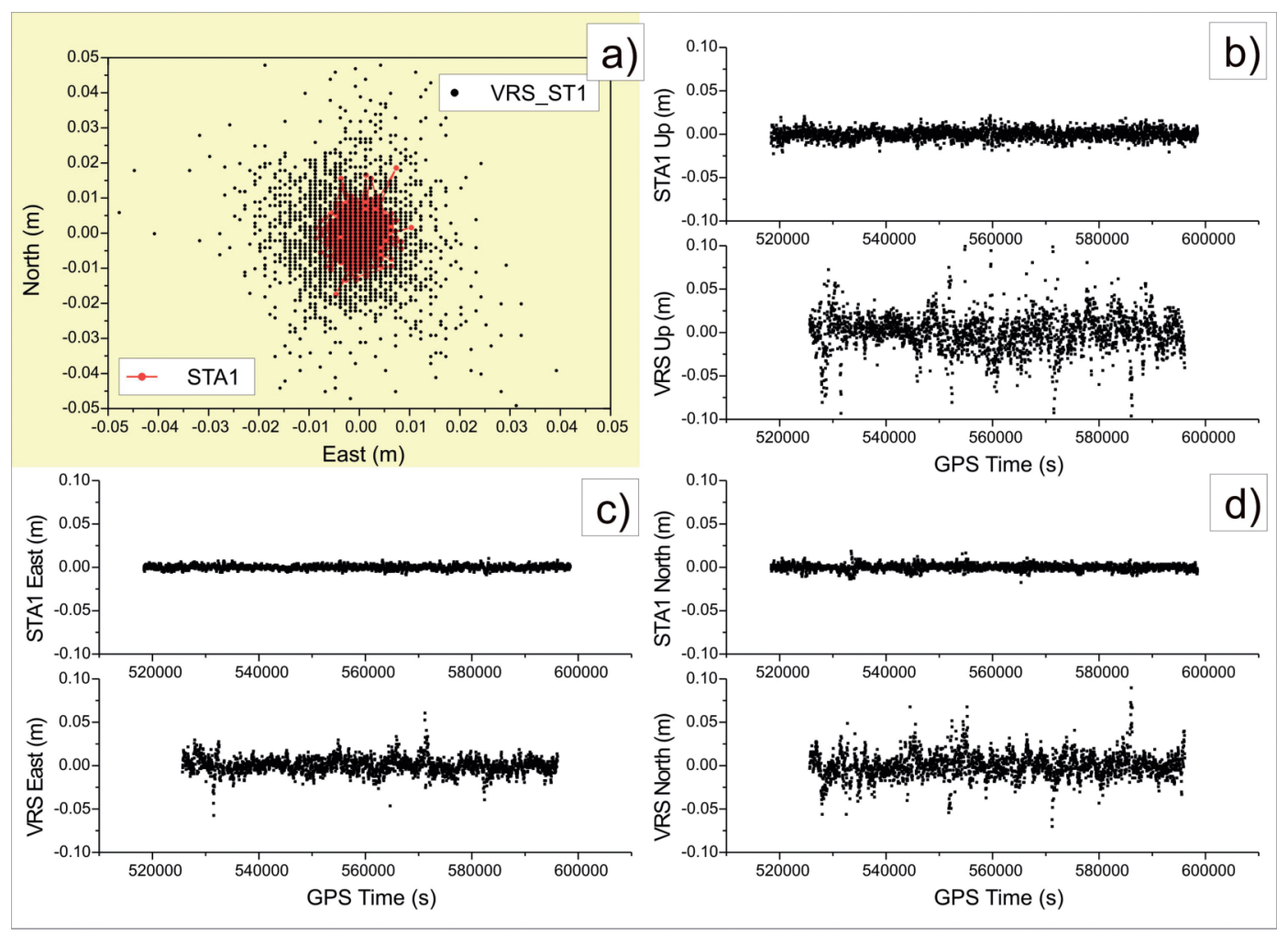

Fig. 2a-d. Rover trajectories and coordinate time series obtained using STA1 or VRS_STA1 as base station; a) the two trajectories are shown with red and black points respectively; b) the Up rover coordinate component time series computed using real and virtual data for the base station; c), d) the east and north coordinate time series of rover, computed with respect to both STA1 and VRS_STA1 base stations.
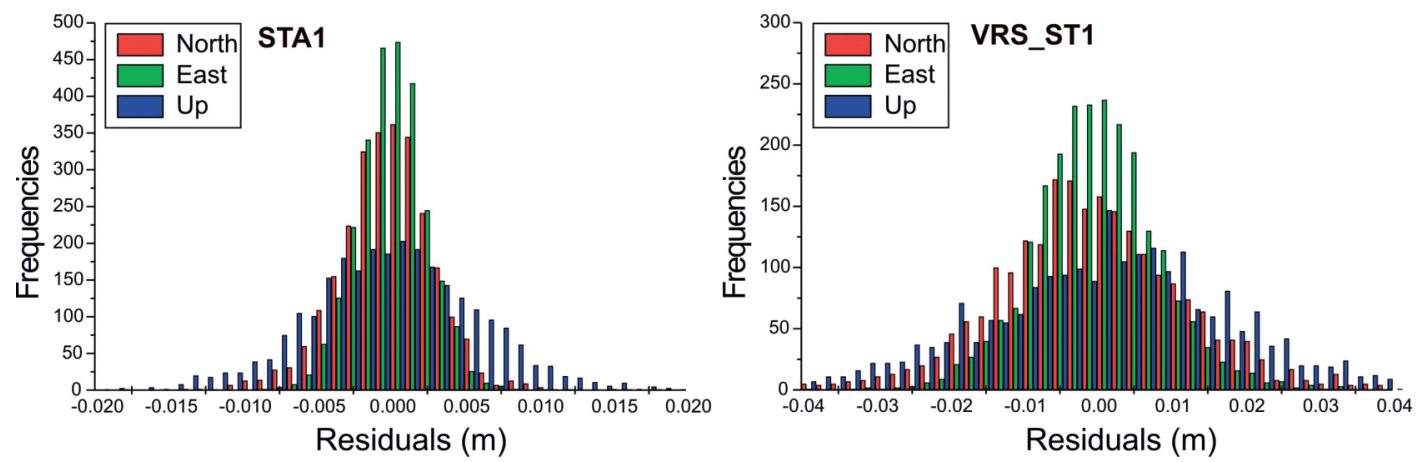

Fig. 3. Rover solutions analysis: statistical distributions of rover coordinates obtained using both STA1 and VRS_STA1 as base stations. 


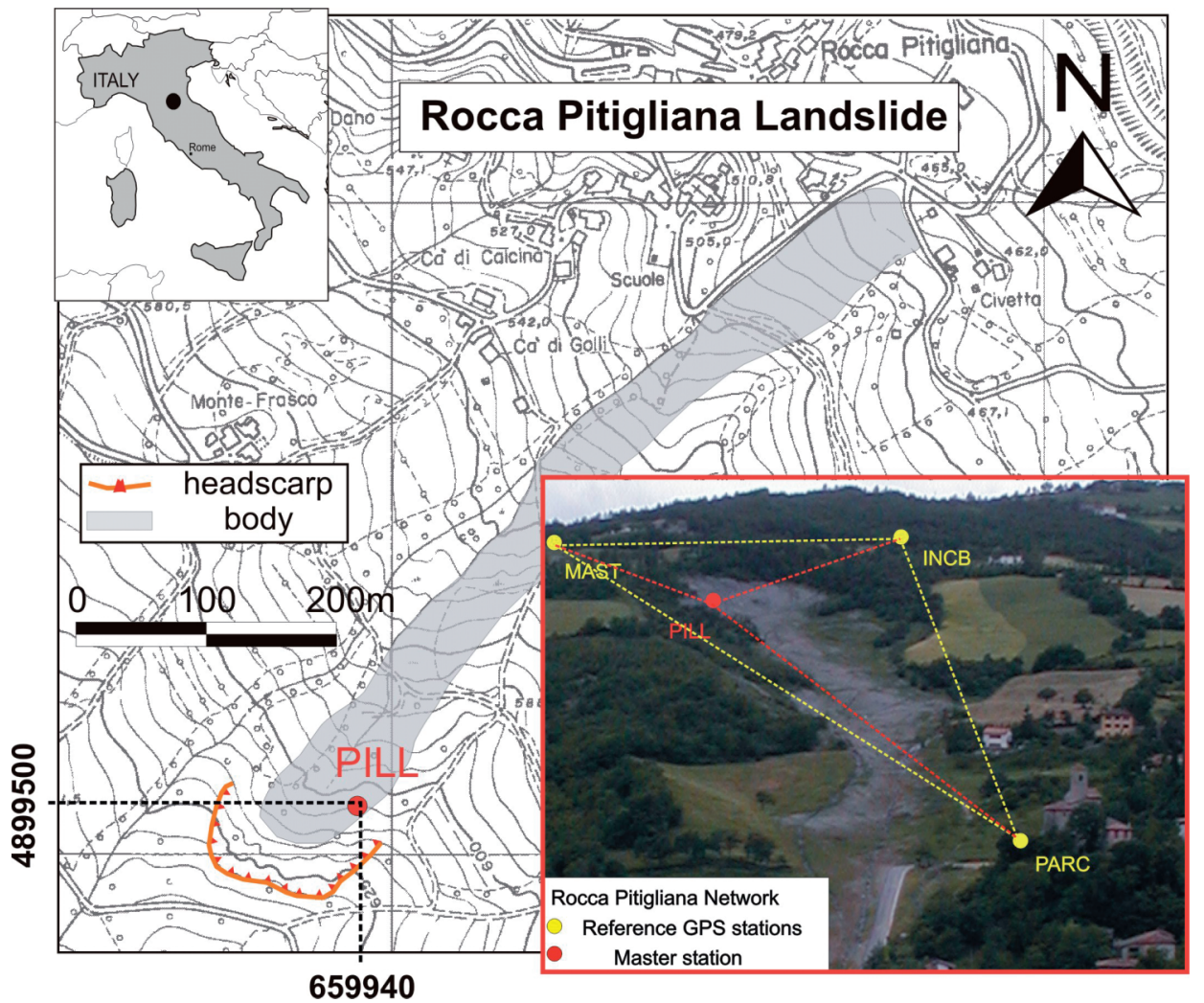

Fig. 4. Rocca Pitigliana Landslide cartography and network description. Three reference stations (yellow circles) are monumented outside the landslide body and one master station (PILL) sits inside the landslide head on a pillar.

errors related to the solutions increase if a VRS is considered, but the reached level is still reasonable for a kinematic measurement session. Moreover, the experiment shows that a VRS does not introduce major systematic effects on computed solutions.

\section{VRS application on landslide surface monitoring}

We applied the VRS approach to the well known and adequately monitored landslide of Rocca Pitigliana (fig. 4) because the performances of this approach could be more easily evaluated together with the corresponding ad- vantages or problems.

The Rocca Pitigliana landslide, located in the Reno River Valley $(50 \mathrm{~km}$ SW Bologna, Italy), is expected to reactivate by means of retrogressive sliding. The material involved in the slope failure is formed by the superficial weathered horizon of stiff and highly fissured mudshales widely outcropping in the Northern Apennines of Italy. It belongs to the Palombini Shale and Varicoloured Shale Formations. The formations are strongly deformed including completely disrupted strata. The assemblage of such highly deformed units has been referred to the literature as «argille scagliose» whose literal translation is scaly clays (Pini, 1999). The first information about its activity 


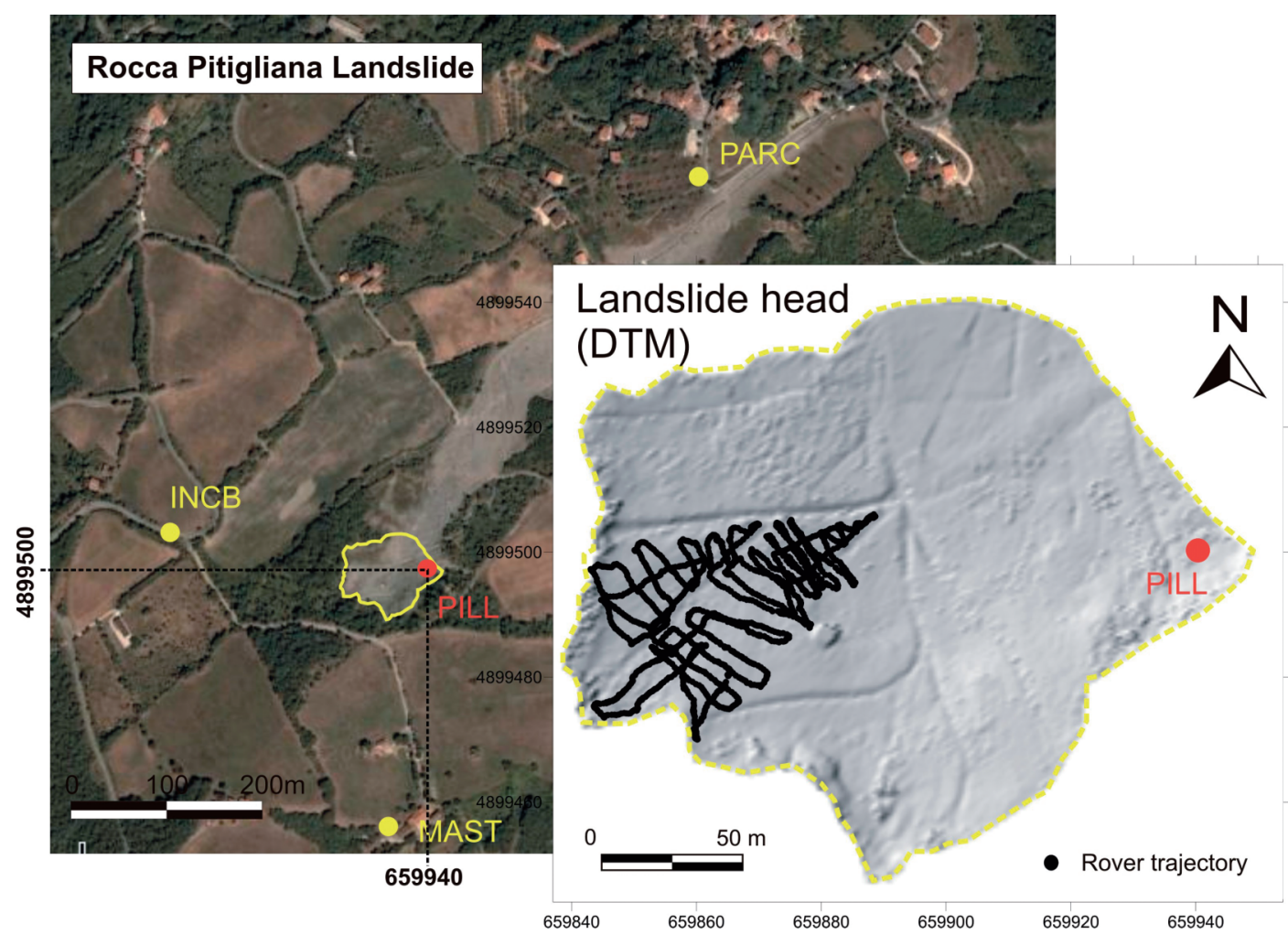

Fig. 5. The rover trajectory is shown (black circles) on a DTM of the landslide head defined in 2005. The yellow dash line points out the boundary of the model also in the aerial image. Yellow circles are the reference stations while the red one is the PILL master station used for kinematic analysis.

refers to March 6th 1934, when several buildings were destroyed. In April 1994 it partially reactivated, while its complete reactivation occurred in 1999, when the main road was interrupted. The reactivations started with small roto-translational movements involving the crown area, subsequently evolving in earthflows.

To allow a continuous monitoring of the unstable mass, two permanent stations were established in past years. The first (MAST) was placed on a rock outcrop outside the landslide area, and therefore stable, whereas the second (PILL) was built on a pillar mounted near the landslide head and affected by landslide movements (fig. 4). 3-D type geodetic monuments were used to assure antenna stability. The other two stations (PARC and INCB) were built on outcropping units and the geodetic monuments were placed in stable areas near the landslide, and used together with MAST and PILL to provide a local reference frame during GPS surveys. The landslide and in particular its upper part was studied using GPS in both static and kinematic modes. Aerial and ground-based photogrammetric surveys were also performed to provide a detailed digital terrain model of the area (Fabris et al., 2005).

In October 2006 a kinematic GPS survey was planned and performed in the Rocca Pitigliana Landslide. The PILL station was started at $1 \mathrm{~s}$ sampling rate and a rover station was moved on the head of the landslide for about 40 minutes. From these data, the rover trajec- 

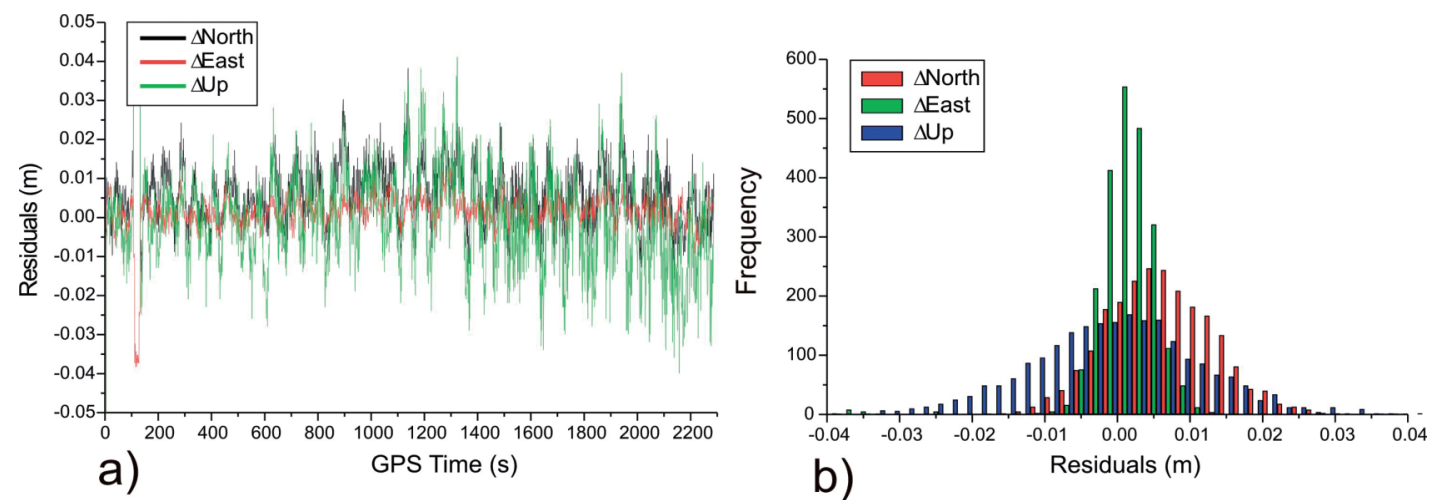

Fig. 6a,b. Real and virtual solution comparison: the two rover coordinate time series are computed with respect to PILL and VRS_PILL base stations; a) residuals obtained for each one of the three coordinate components by directly subtracting the real and virtual rover solution; b) statistical distribution of residuals.

tory was computed in post-processing kinematic mode using the procedure described in the previous chapter. In particular, the PILL coordinates were maintained fixed to the values provided by the analysis of the reference network consisting in MAST, INCB and PARC stations. Results of such computations are shown in fig. 5, where rover kinematic coordinates are overimposed on a Digital Terrain Model (DTM) obtained by the integration of terrestrial laser scanner and GPS measurements, previously carried out (Pesci et al., 2006).

The kinematic post-processing was repeated considering the VRS_PILL virtual station. The RINEX file of this virtual station was generated from the previously computed PILL coordinates and the data of the reference network (MAST, INCB and PARC). Also in this case the rover trajectory is modeled, clearly using the same observational rover data of the previous case. The differences of coordinates obtained using PILL and VRS_PILL were computed; the corresponding residuals are shown in fig. 6 . The results agree within the solution accuracy. In particular, the standard deviation for the three components of coordinate residuals were $0.013 \mathrm{~m}$ (North component), $0.010 \mathrm{~m}$ (East component) and $0.023 \mathrm{~m}$ (elevation) respectively.

\section{Discussion and conclusions}

The Assogeo reference network consists of several permanent stations homogeneously distributed in the Emilia-Romagna and surrounding regions, allowing the generation of stable synthetic RINEX files at known and chosen coordinate points. A virtual station is created at a defined coordinate point using GPS data of surrounding permanent stations; the closer these stations are, the lower the noise of virtual station data obtained from interpolation/extrapolation procedures is.

A GPS network of permanent stations distributed on an irregular grid with a mean step of a few tens of kilometers is sufficient in order to obtain a set of synthetic observables characterized by a few centimeters' noise. By choosing a suitable grid step, it is possible to create data for some GPS-based applications to survey and study surface kinematics at a small scale (e.g. landslide).

The aim of the described research was to test the stability and accuracy of solutions obtained from post-processing analysis of GPS data when a VRS is considered.

The experiment performed on the roof of the Physics Department (Bologna University) is conceived to estimate the noise affecting the final coordinate time series when a VRS is used 
instead of a real station. In this case, the VRS is generated from a surrounding network with a size of about $50 \mathrm{~km}$, and the obtained results are very significant. The standard deviations of the solutions are about $0.011 \mathrm{~m}$ for the horizontal and $0.025 \mathrm{~m}$ for the vertical component, about two or three times the values which can be obtained using a real data set. Despite a larger noise and dispersion in the VRS case, the resulting point coordinates are substantially the same, since the differences are of a few millimeters. Therefore, the use of a VRL does not introduce any significant systematic effects.

Another experiment was performed using data provided by measurement sessions at the Rocca di Pitigliana landslide, located in the Bologna Apennines (about $50 \mathrm{~km}$ from the city of Bologna). This site is a particularly suitable case study because a reference GPS network is available here, which consists of three GPS stations located on stable outcrops near the landslide body. In this way, the occupations of points of known coordinates was possible, and the measurements were carried out on a well measured physical surface.

This experiment is very similar to the first one, but in this case the aim was to evaluate the effect of VRS introduction on the results of the rover trajectory post-processing. Another difference between the two experiments is due to the fact that the VRS is now introduced by the processing of data related to GPS receivers at distances shorter than $1 \mathrm{~km}$. The experimental data were obtained by walking on the disrupted head of the landslide for about 40 minutes. A comparison between real and virtual processed trajectories, operated taking into account the coordinate differences on the same epochs, shows that results agree. In particular, the standard deviations of the differences are about $0.01 \mathrm{~m}$ for the horizontal and $0.02 \mathrm{~m}$ for the vertical components, without the detection of any systematic effects.

Results, obtained in different environments and different surveys and post-processing modes (static and kinematic), show that a VRSbased approach is sufficient to provide high accuracy data for the studied surface. In particular, our results confirm that the existence of a permanent network allows the generation of a
VRS and a fast and rapid post-processing, avoiding the use of several GPS receivers, saving time and costs. A traditional kinematic acquisition of a landslide surface requires a GPS base station and a rover station carried on a backpack by an operator, walking on the investigated surface. If a VRS is used, the base station (PILL in the described experiment) is not necessary. Therefore, the measurements can be performed using only one GPS receiver at lower costs. On the other hand, if two receivers are available, both can be used to track different trajectories on the landslide surface.

The data provided by a GPS kinematic measurement session on a landslide surface can be used to produce a digital terrain model (DTM) of the unstable slope surface, with an accuracy of 0.1-0.2 $\mathrm{m}$ and a resolution of 0.5 $1.0 \mathrm{~m}$. A comparison between two or more multi-temporal DTM models can detect possible variations of the observed scene, e.g. differences of elevations, as well as the computation of the displacement field. Nevertheless, when a VRS is used instead of a real master station, the errors are 2-3 times larger. For this reason, the computation of some quantities, for example the strain, requires adequate care. In particular, the strain computation is generally based on least square (LS) solution of a series of normal equations, where a design matrix is considered (Pesci and Teza, 2007; Teza et al., 2007). This matrix, which describes the position of the experimental points with respect to the points where the strain computation is performed, is considered to have negligible errors (the uncertainties are related to the pseudo-observable vector only, which is the vector formed by displacements of the experimental points). If data are provided by VRS-based measurements, the design matrix errors could be significant and the use of a total LS approach could be necessary to obtain reliable results (Golub and Van Loan, 1980). A development of the method presented in Pesci and Teza, 2007 and in Teza et al., 2007, based on incorporation in this method of the total LS approach, is in progress.

A further development concerns the use of VRS stations to create a stable and time-independent reference network in the surroundings of the interested area. In particular, a possible 
definition of an optimal network geometry for an assigned landslide, based on both real and virtual stations, is considered and will be developed and tested. These experiments point out a general validity of an approach to landslide monitoring based on systematic use of virtual stations.

Nowadays, the introduction of a VRS is often used when a GPS network is available but none of the stations of this network are located near the area where the measurements are carried out. This is the case of a regional scale network whose mean spacing between stations is $50-60 \mathrm{~km}$, and there is a lack of points within some tens of kilometers around the surveying area, or at a landslide scale, spaced by a hundred or a thousand meters, without reference points in its surroundings. In Italy, some Public Authorities, in particular some Regions, and also private companies have placed or have planned to place good networks of permanent GPS stations; the mean spacing is therefore reduced to tens of kilometers. Nevertheless, also in such a case the use of a VRS could provide some benefits. Results of these experiments show that the method used provides reasonable solutions in all the explored cases. An important statement is that the standard deviations are increased by a factor of 2 or 3 with respect to a case where a real station is used, without any valuable systematic effect. In particular, the performance is significantly improved with respect to the classical approach where the user processes data related to the nearest permanent station, and no distortions are introduced.

A very important practical indication arises from our experiments: in the case of landslide monitoring, a kinematic survey can be carried out without a master station and therefore using only one rover receiver carried by an operator walking on the slope surface (cost saving case), or two rovers, both carried on the slope by two operators (time saving case).

In conclusion, a VRS approach to GPSbased topographic measurements can lead to significant benefits in landslide monitoring in those areas where either a thick network of permanent stations is not present, or data with suitable sampling rate (e.g. $1 \mathrm{~s}$ in the case of a kinematic survey) are unavailable.

\section{Acknowledgments}

The authors to thank the ASSOGEO company for software, instruments, GPS data of permanent stations and for their strong collaboration. Special thanks to Luca Gusella for his assistance during the kinematic landslide surveying and to Paolo Baldi for scientific support. Finally many thanks to Lydia Gulick for the help in translating and correcting the text.

\section{REFERENCES}

Achilli, V., P. Baldi, L. Baratin, C. Bonini, E. Ercolani, S. Gandolfi, M. Anzidei and F. Riguzzi (1997): Digital photogrammetric survey on the island of Vulcano. Acta Vulcanologica, 9 (2), 1-5.

Anzidei, M., P. Baldi, A. Pesci, A. Esposito, A. Galvani, F. Loddo, P. CRistofoletti, A. MassucCi and S. Del Mese (2005): Geodetic deformation across the central apennines from gps data in the time span 1999-2003, Annals of Geophysics, 48 (2), 259-271.

Anzidei, M., G. Casula, A. Galvani, F. Riguzzi, G. Pietrantonio, E. Serpelloni, A. Esposito, A. Pesci, F. Loddo, A. Massucci and S. Del Mese (2006): Le prime stazioni GPS permanenti INGV-CNT per il monitoraggio delle deformazioni crostali nell'area italiana, Quaderni di Geofisica, 39, 1-46.

Baldi, P., N. Cenni, M. Fabris and A. ZanutTa (2008): Kinematics of a landslide derived from archival photogrammetry and GPS data, Geomorphology, 102, 435-444.

Baldi, P., S. Bonvalot, P. Briole and M. Marsella (2000): Digital photogrammetry and kinematic GPS for monitoring volcanic areas, Geophysics Journal International, 142 (3), 801-811.

Beutler, G., G.W. Hein, W.G. Melbourne and G. Seeber (1995): GPS Trends in Precise Terrestrial, Airborne, and Spaceborn Applications, International Association of Geodesy Symposia, 115, 275-338.

Bonforte, A., M. Anzidei, G. Puglisi, M. Mattia, O. Campisi, G. Casula, A. Galvani, A. Pesci, B. Puglisi, F. GREstA and P. BALDI (2002): Gps surveys in the foreland-foredeep tectonic system of Southeastern Sicily: first results, Annals of Geophysics, 45, 673682.

Cenni, N., P. Baldi, E. Mantovani, M. Ferrini, M. Viti, V. D'Intinosante, D. BabBucci and D. Albarello (2008): Short-term (geodetic) and long-term (geological ) deformation pattern in the Northern Apennines. Bollettino della Società Geologica Italiana, 127, (1).

FABRIS, M. and A. PESCI (2005): Automated DEM extraction in digital aerial photogrammetry: precisions and validation for mass movement monitoring, Annals of Geophysics, 48, 973-988.

GILI, J.A., J. CoRominas and J. RiUs (2000): Using Global Positioning System techniques in landslide monitoring, Engineering Geology, 55, 167-192.

Golub, H.G. and F.C. VAN LOAN (1980): An analysis of the total least square method, SIAM Journal of Numerical 
Analysis, 17 (6), 883-893.

GURTNER, W. (1994): RINEX - the receiver-independent exchange format, GPS World, 5 (7), 48-52.

HÄKLI, P. (2004): Practical test on accuracy and usability of Virtual Reference Station method in Finland, FIG Working Week 2004, Conference Proceedings, Athens, Greece, May 22-27.

Higgins, M.B. (2002): Australia's changing surveying infrastructure from marks in the ground to virtual reference stations, Proceedings of FIG XXII International Congress, April 19-26, (Washington, USA, CD-ROM paper TS5.6).

Hofman-Wellenhof, B., H. Lichtenegger and J. Collins (1997): GPS Theory and Practice, (Springer Verlag Wien, New York), pp. 214-258.

Hu G.R., H.S. KHOO, P.C. GoH and C.L. LAW (2003): Development and assessment of virtual reference stations for RTK positioning, Journal of Geodesy, 77 (5-6), 292-302.

Hu G.R., D.A. Abbey, N. Castleden, W.E. Featherstone, C.J. Earls, O. Ovstedal and D. Weiring (2005): An approach for instantaneous ambiguity resolution for medium- to longrange multiple reference station networks, GPS Solutions, 9 (1), 1-11.

Hunstad, I., M. Anzidei, M. Cocco, P. Baldi, A. Galvani and A. Pesci (1999): Modelling coseismic displacements during the 1997 Umbria-Marche earthquake (Central Italy), Geophysical Journal International, 139, 283-295.

Kouba J. and P. Héroux (2001): Precise Point Positioning using IGS orbit and clock products, GPS Solutions, 5 (2), 12-28.

LANDAU, H. and H.J. EULER (1992): On-the-fly ambiguity resolution for precise differential positioning. Proc. 5th Int. Tech. Meeting of the Satellite Division of the U.S. Inst. of Navigation, 16-18 September, 1992, pp. 607-613.

Mora P., P. Baldi, G. Casula, M. Fabris, M. Ghirotti, E. Mazzini and A. Pesci (2003): Global Positioning Systems and digital photogrammetry for the monitoring of mass movements: application to the Ca' di Malta Landslide (northern Apennines, Italy), Engineering Geology, 68, 103-121.

Moss, J. (2000): Using the Global Positioning System to monitor dynamic ground deformation networks on potentially active landslides, International Journal of Applied Earth Observation and Geoinformation, 2 (1), 24-32.

Pesci, A., P. Baldi, A. Bedin, G. Casula, N. Cenni, M. FabRIS, F. Loddo, P. MoRA and M. BACCHETTI (2004): Digital elevation models for landslide evolution monitoring: application on two areas located in the Reno River Valley (Italy), Annals of Geophysics, 47, 1339-1353.

Pesci, A., P. Baldi, A. Bedin, G. Casula, N. Cenni, M. FabRIS, F. LODDO, P. MoRA and M. BACCHETTI (2005): Digital elevation models for landslide evolution monitoring: application on two areas located in the Reno River Val- ley (Italy), Annals of Geophysics, 47, 1339-1353.

Pesci, A., D. Conforti, M. FAbris and F. Loddo (2006): GPS, fotogrammetria digitale e laser a scansione: un sistema multidisciplinare e multitemporale di rilevamento, Quaderni di Geofisica, 40, 2006.

Pesci, A. and G. TezA (2007): Strain rate computation, results validation and application: the kinematics of Central Apennines from GPS velocities, Bollettino di Geodesia e Scienze Affini, 66 (2), 69-88.

Pietrantonio, G. and F. RiguzZi (2004): Three-dimensional strain tensor estimation by GPS observations: methodological aspects and geophysical applications, Journal of Geodynamics, 38 (1), 1-18.

PINI, G.A. (1999): Tectonosomes and Olistostromes in the Argille Scagliose of the northern Apennines, Italy. Geological Society of America Bullettin, Special Paper 335, pp. 73.

RIzOS, C. and S. HAN (1998): Status and trends for high precision GPS kinematic positioning, 9th Australasian Remote Sensing \& Photogrammetry Conference, (Sydney, Australia, 20-24 July, CDROM, paper 49), pp. 17.

Serpelloni, E., M. Anzidei, P. Baldi, G. Casula, A. GalVANI, A. PESCI and F. RiguzZI (2001): Geodetic deformations in the central southern Apennines (Italy) from repeated gps surveys, Annali di Geofisica, 44, 627647.

Squarzoni, C., C. Delacourt and P. Allemand (2005): Differential single-frequency GPS monitoring of the La Valette landslide (French Alps), Engineering Geology, 79 (3-4), 215-229.

Teza, G., A. Pesci., R. Genevois and A. Galgaro (2007): Characterization of landslide ground surface kinematics from terrestrial laser scanning and strain field computation, Geomorphology, 97 (3-4), 424-437.

Trimble (1999): Trimble Navigation Limited, Guide to the Trimble Geomatics Office Sample Data, (Trimble Navigation, Sunnyvale, CA).

Tzenkov, T. and S. Gospodinov (2003): Geometric analysis of geodetic data for investigation of 3D landslide deformations, Natural Hazard Review, 4 (2), 78-81.

Vespe, F., G. Bianco, M. Fermi, C. Ferraro, A. Nardi and C. SCIARRETTA (2000): The Italian GPS Fiducial Network: Services and Products, Journal of Geodynamics, 30, 327-336.

Vollath, U., A. Buecherl, H. Landau, C. Pagels and B. WAGNER (2000): Multi-base RTK positioning using virtual referencestations, Proceedings of ION GPS-2000, (19-22 Sept, Salt Lake City, Utah, USA), 123-131.

WANNINGER, L. (2002): Virtual reference stations for centimetre level kinematic positioning, Proceedings of ION GPS- 2002, (24-27 Sept, Portland, Oregon, USA), 1400-1407.

(received December 17, 2007; accepted May 12, 2008) 